\title{
Pierre Reverdy, Les Ardoises du toit (Paris, Paul Birault, 1918), exemplaire corrigé par l'auteur
}

\section{Rudolf Mahrer et Christophe Imperiali}

\section{(2) OpenEdition}

1 Journals

\section{Édition électronique}

URL : https://journals.openedition.org/genesis/1783

DOI : 10.4000/genesis. 1783

ISSN : 2268-1590

Éditeur :

Presses universitaires de Paris Sorbonne (PUPS), Société internationale de génétique artistique littéraire et scientifique (SIGALES)

Édition imprimée

Date de publication : 9 mai 2017

Pagination : 165-180

ISBN : 979-1023-105636

ISSN : 1167-5101

\section{Référence électronique}

Rudolf Mahrer et Christophe Imperiali, «Pierre Reverdy, Les Ardoises du toit (Paris, Paul Birault, 1918), exemplaire corrigé par l'auteur », Genesis [En ligne], 44 | 2017, mis en ligne le 18 mai 2018, consulté le 17 mars 2023. URL : http://journals.openedition.org/genesis/1783 ; DOl : https://doi.org/10.4000/ genesis. 1783 


\title{
" "Pourquoi fais-tu ça ?"... "Et si tu faisais autrement !" ” Pierre Reverdy, Les Ardoises du toit (Paris, Paul Birault, 1918), exemplaire corrigé par l'auteur
}

\author{
Présenté par Christophe Imperiali et Rudolf Mahrer
}

$\mathrm{C}$ 'est par ces mots que Pierre Reverdy taraudait son ami Gino Severini tandis qu'il l'observait peindre 1 . Ce penchant réflexif, qu'il appelait esthétique plutôt que théorie, Reverdy n'y a pas seulement donné cours dans ses importantes contributions à l'histoire de l'art de son temps : sa propre écriture en porte la trace. C'est du moins ce que jugera le lecteur de cet exemplaire de l'édition originale des Ardoises du toit corrigé par le poète et que conservent les archives de la Bibliothèque littéraire Jacques Doucet ${ }^{2}$. On y observe en effet un trajet qui semble, pour certains textes au moins, annihiler si bien ce que l'on considère comme la manière propre du poète qu'on en vient à se demander à quel titre il s'agit encore des mêmes poèmes et de cette même manière. Le vertige de la totale virtualité, caractéristique des premiers moments de l'avant-texte - où l'on peut faire «ça» ou faire «autrement»-, Reverdy l'éprouve et l'exploite encore «après le texte».

\section{Datation}

À quelle période convient-il de situer la campagne de réécritures dont l'exemplaire d'auteur des Ardoises du toit garde témoignage ? Le terminus a quo est évidemment donné par la date du support : l'édition originale, fabriquée à Paris par Paul Birault en une centaine d'exemplaires, a paru le 15 mars 1918. Quant au terminus ad quem, il est à placer en 1924. On remarque en effet des croix, au crayon gris ou bleu, dans le haut de certaines pages de l'exemplaire corrigé : elles signalent les poèmes sélectionnés pour la publication, aux éditions de la NRF, des Épaves du ciel, en juin 1924. Le volume réunit deux cent quatre-vingt-deux poèmes tirés des premiers recueils de Reverdy. Il se révèle étonnamment sélectif à l'endroit de deux d'entre eux : Les Ardoises du toit justement, dont ne sont repris que quarante-cinq des quatre-vingt-un poèmes originaux, et La Guitare endormie (Paris, Paul Birault, 1919), dont ne figurent que cinq textes sur trente-huit. Pourquoi un tel écrémage? Sans doute parce qu'en 1924, il est déjà prévu qu'un autre recueil de Reverdy rejoigne la série «Une œuvre, un portrait» des Éditions de la NRF. Il s'agit d'Écumes de la mer (1925): le portrait est de Picasso et l'œuvre se compose précisément de soixante-six pièces des Ardoises du toit et de La Guitare endormie écartées des Épaves du ciel. Le procédé évite ainsi de rééditer une partie des mêmes textes à une année d'intervalle ${ }^{3}$ (voir tableau 1).

Les réécritures observables sur l'édition originale des Ardoises du toit datent ainsi manifestement d'une même campagne de réécritures préparant, entre 1920 et 1924, Les Épaves du ciel et Écumes de la mer. Cette préparation est complète : le dernier état textuel porté par l'exemplaire corrigé coïncide avec celui des deux éditions de 1924 et de 1925. C'est pourquoi il nous est possible de donner, en dessous des poèmes crayonnés de l'édition originale, les pages correspondantes des Épaves du ciel ou des Écumes

1. Du moins est-ce ainsi que le peintre se rappelle les débuts de son amitié avec Reverdy dans Pierre Reverdy, 1889-1960, Paris, Mercure de France, 1962 (cité par Étienne-Alain Hubert, «Introduction », Pierre Reverdy, Euvres complètes, t. I, Paris, Flammarion, 2010, p. XVII).

2. Remercions ici la Bibliothèque littéraire Jacques Doucet pour son accueil éclairé et François Chapon, président du Comité Reverdy, pour nous avoir accordé le droit de la consultation puis celui de la reproduction partielle du document que nous présentons ici.

3. L'exemplaire corrigé de La Guitare endormie est également conservé à la Bibliothèque littéraire Jacques Doucet. À propos de ces quelques éléments exogénétiques, voir la notice de Plupart du temps par ÉtienneAlain Hubert dans Pierre Reverdy, Euvres complètes, op. cit., p. 12791286. Nous remercions ici Olivier Gallet qui nous a permis de préciser notre situation succincte du document - toute approximation restant nôtre. 


\begin{tabular}{l|c|c|c|c|}
\cline { 2 - 5 } & $\begin{array}{c}\text { Édition originale } \\
(1918)^{*}\end{array}$ & $\begin{array}{c}\text { dans Les Épaves du ciel } \\
(1924)\end{array}$ & $\begin{array}{c}\text { dans Écumes de la mer } \\
(1925)\end{array}$ & $\begin{array}{c}\text { dans Plupart du temps } \\
(1945)\end{array}$ \\
\hline Visite & {$[38 \mathrm{~g}]$} & & p. 27 & p. 223 \\
\hline Manchette & {$[38 \mathrm{~d}]$} & & & p. 224 \\
\hline Couvre-feu & {$[39 \mathrm{~g}]$} & p. 100 & & p. 225 \\
\hline Entre deux mondes & {$[39 \mathrm{~d}]$} & & p. 28 & p. 228 \\
\hline Campagne & {$[41 \mathrm{~d}]$} & p. 134 & & p. 229 \\
\hline Patience & {$[42 \mathrm{~g}]$} & & p. $30-31$ & p. 230 \\
\hline Visage & {$[42 \mathrm{~d}]$} & & p. 32 & p. 233 \\
\hline Course & {$[44 \mathrm{~g}]$} & & p. 35 & p. 234 \\
\hline Étape & {$[44 \mathrm{~d}]$} & & p. $36-37$ & p. 235 \\
\hline Écran & {$[45 \mathrm{~g}]$} & p. 104 & & p. 236 \\
\hline Et là & {$[45 \mathrm{~d}]$} & p. 116 & & \\
\hline
\end{tabular}

(*) Édition non paginée; dans l'exemplaire corrigé, une foliotation est portée au crayon gris en haut à gauche de chaque double page. Nous nous y référons en précisant si le poème se situe à gauche ou à droite de la double page.

Tableau 1 : Les poèmes présentés ici, d'une édition à l'autre des Ardoises du toit

de la mer en guise de transcription linéaire - bien que drôlement linéarisée.

Vingt ans après, Plupart du temps. Poèmes 1915-1922 regroupera, dans sa section intitulée «Les Ardoises du toit», les poèmes du recueil original de 1918, à l'exception de «Manchette», déjà exclu des deux éditions intermédiaires. La comparaison des versions publiées dans Plupart du temps avec celles des éditions antérieures de 1924 et 1925 ne fait pas apparaître de variations «segmentales ». L'auteur n'a plus modifié la lettre de ses poèmes. On observe en revanche, sur le plan «suprasegmental», celui de la topographie des textes, de légères mais fréquentes modifications. À cet égard, «Visage» fait figure d'exception (p. 174).

\section{Créer selon l'objet ou la poïétique du texte à trous}

Ce qui ne laisse pas de frapper l'attention, en particulier lorsque l'on considère les six collages qui parsèment l'exemplaire d'auteur, c'est la manière dont l'écrivain «ruse » (comme dit Michel Jeanneret, cité p. 23) pour transformer l'espace du livre, lieu du texte, en espace de l'invention. Reverdy pense avec ou selon la matérialité qui s'offre à lui dans cette phase particulière de la création. Prenons «Couvre-feu» ou «Visage» (p. 171 et p. 174); ils présentent de nombreux ajouts sans aucun becquet d'insertion : le blanc de l'édition originale - auquel étaient confiées les relations entre groupes, ou la modulation du sens d'un constituant isolé sur la page - accueille l'ajout (d'un verbe, d'un adverbe ou d'un syntagme à fonction circonstancielle), explicitant notamment la relation ou la modulation. Dans l'exemplaire corrigé, l'espace de la signification est devenu espace de la création, selon un déroutant geste d'écriture qui revient à compléter un texte à trous.

\section{Les Ardoises du toit de 1918 dans leur contexte esthétique}

En 1918, Les Ardoises du toit paraissent ornées de deux dessins de Braque. La même année, les Calligrammes sont assortis d'un portrait d'Apollinaire par Picasso et, l'année suivante, les Dix-neuf poèmes élastiques de Cendrars seront accompagnés d'un portrait de l'auteur par Modigliani. La modernité poétique se tourne résolument vers les arts plastiques. En plus d'être illustrés par les peintres les plus en vue, ces trois recueils sont aussi remarquables par les innovations typographiques qu'ils mettent en œuvre. La proposition de Reverdy, dans ce contexte, est pourtant originale et singulière, comme il le relève lui-même en 1919 dans Self defence :

Tandis que d'autres pratiquaient des dispositions typographiques dont les formes plastiques introduisaient en littérature un élément étranger, apportant d'ailleurs une difficulté de lecture déplorable, je me créais une disposition dont la raison d'être purement littéraire était la nouveauté des rythmes, une indication plus claire pour la lecture, enfin une ponctuation nouvelle, l'ancienne ayant peu à peu disparu par inutilité de 
mes poèmes. Cette disposition répondait en même temps au besoin de remplir par l'ensemble nouveau la page qui choquait l'œil depuis que les poèmes en vers libres en avaient fait un cadre asymétriquement rempli 4 .

À la fin de 1919, Reverdy paraît ainsi bien convaincu de la pertinence du dispositif qu'il a inauguré. Aujourd'hui encore, d'ailleurs, la critique reverdyenne est à peu près unanime à considérer la typographie des Ardoises comme l'une des grandes trouvailles du poète, et la marque la plus originale de son style propre.

Et pourtant, l'adhésion de Reverdy à ce type de dispositifs sera de courte durée. Bien plus tard, il exprimera ainsi à son ami François Chapon la raison qui l'avait conduit à retoucher ses poèmes de la fin des années dix : «Je m’étais aperçu à temps que presque toute la substance restait en moi, par excès de pudeur, et que j'aboutissais au dessèchement ${ }^{5}$.» Face à un tel constat, le poète aurait alors décidé de mettre « un peu de chair sur le squelette ${ }^{6}$ » que constituaient ses textes anciens. En 1924, il décrit le travail dont témoigne notamment notre exemplaire corrigé : «je n'ai eu ces dernières années qu'un certain plaisir au milieu de beaucoup de déboires intellectuels, c'est de pouvoir rectifier la plupart des poèmes des Ardoises du toit et de La Guitare endormie. Je ne peux juger avec certitude que je les ai améliorés, mais j'ai obtenu ainsi qu'ils me dégoûtent moins pour le moment ${ }^{7}$.»

\section{Que reste-t-il des Ardoises de 1918?}

Ces citations sont importantes pour appréhender le travail de Reverdy sur les Ardoises. Elles indiquent tout à la fois un changement d'esthétique très net entre 1919 et 1924 , mais aussi, de façon surprenante, un désir de «rectifier» des textes antérieurs pour leur donner une seconde vie. En général, quand un poète est «dégoûté» par ses poèmes anciens, il en écrit de nouveaux, et le changement d'esthétique est perceptible à la comparaison de deux recueils distincts. Pourquoi donc Reverdy juge-t-il bon de revenir sur ses poèmes de 1918 ? Et pourquoi considère-t-il ce travail comme la seule satisfaction de ces années?

Ces questions en engagent une autre, que la nature du travail pratiqué sur ces poèmes force à se poser : dans quelle mesure les poèmes retouchés restent-ils les mêmes poèmes que ceux qu'ils étaient précédemment? Dans Plupart du temps, en 1945, Reverdy indique pour la partie «Les Ardoises du toit» la date de 1918. Mais que reste-t-il dans Plupart du temps des textes de 1918 ? Il reste les titres : si l'on excepte le poème supprimé («Manchette») et un titre modifié («Heure d'été » qui devient «Montre»), la table des matières des deux recueils est exactement identique ${ }^{8}$. Il paraît donc logique, sur cette base, d'estimer qu'il s'agit bien d'un seul et même recueil et que les modifications apportées aux poèmes sont de l'ordre de la variation du même et non de la transformation en autre chose.

Pourtant, lorsqu' on se penche de plus près sur ces modifications, on ne peut que se demander où réside foncièrement cette supposée identité. Cette question se pose plus ou moins impérieusement selon les poèmes : pour un quart d'entre eux environ, qui sont repris sans modification ${ }^{9}$, elle ne se pose pas. Mais pour la plupart des poèmes, les modifications sont non seulement conséquentes, mais surtout réparties de façon étonnamment généreuse sur l'ensemble des paramètres de l'écriture poétique.

\section{Chemins de la réécriture : l'exemple de «Campagne»}

Pour prendre la mesure de ces variations, regardons de plus près le cas de «Campagne» (p. 172-173). Ce poème est particulièrement intéressant d'un point de vue génétique parce qu'aux retouches apportées sur l'original s'ajoute un papillon lui-même raturé et corrigé. Mais la nature et l'ampleur des modifications apportées n'en fait pas un cas exceptionnel : un grand nombre d'autres poèmes permettraient d'observer les mêmes phénomènes.

4. Self defence, dans Euvres complètes, op. cit., p. 530-531.

5. Cité dans Étienne-Alain Hubert, «Introduction», op. cit., p. XXVIII.

6. Idem.

7. «Fragments incorporés à "Pierre Reverdy m'a dit" de Benjamin Péret», dans CEuvres complètes, op. cit., p. 595-596.

8. Signalons, à ce propos, une erreur dans la reproduction en facsimilé de l'édition des CEuvres complètes chez Flammarion : «Projets» a été omis, et «La Saison dernière » remonté de deux rangs dans l'ordre des poèmes. 9. C'est le cas de vingt-deux poèmes, dont le matériau lexical ne change pas. Pour deux d'entre eux, quelques modifications typographiques interviennent, mais vingt demeurent aussi inchangés sur ce plan. 
Sur le plan du matériau verbal, on constate que sur les cent quatorze mots que compte la version définitive 10 , seuls cinquante-cinq sont empruntés au poème de 1918, qui en comptait soixante-dix-neuf. Autrement dit, Reverdy réemploie $70 \%$ des mots du poème initial, qui forment $48 \%$ du poème final. Plus de la moitié des mots sont donc nouveaux dans cette nouvelle «Campagne», conformément à une tendance générale qui consiste à reprendre une partie du texte antérieur et à l'étoffer. Reverdy, on l'a vu, parlait de mettre de la chair sur un squelette : certaines pages du livre corrigé illustrent cette métaphore, avec le texte imprimé chargé d'insertions au crayon de toutes parts. Mais pourquoi ces ajouts? De quelle «chair» s'agit-il ? On identifiera aisément deux types d'ajouts, situés sur deux plans distincts : la syntaxe et la métrique.

Du côté de la syntaxe s'observe une tendance très nette à l'explicitation et au liage de ce qui restait flottant dans les textes de 1918. Les exemples sont si nombreux et si patents qu'il n'est pas utile d'insister; disons simplement que ces retouches rompent avec ce que Reverdy présentait lui-même, en 1918-1919, comme un renouvellement de la syntaxe, par lequel la disposition typographique suppléait partiellement aux liens grammaticaux ${ }^{11}$. En développant les liens syntaxiques et en explicitant les articulations logiques de son texte, Reverdy revient assurément à une écriture plus traditionnelle que celle qui caractérisait ses poèmes de 1918.

C'est la même impression d'un retour à quelque chose de plus classique qui se dégage des ajouts motivés par la métrique. Dans «Campagne», en 1918, on trouve des vers de neuf quantités syllabiques différentes, allant de deux à douze syllabes; la moitié d'entre eux sont impairs et la majorité (14/18) inférieurs à huit syllabes. Dans la version retouchée, on ne retrouve plus que deux mètres : 1'octosyllabe 12 et l'alexandrin; plus de vers bref ni de vers impair; tous les vers inférieurs à huit syllabes ont été allongés pour atteindre cette mesure, qui devient très largement dominante dans l'ensemble des poèmes retouchés. Il faut ajouter que le nombre de rimes augmente également et que leur disposition devient plus prévisible : dans la version finale, seize vers sur dix-sept riment, et si l'on retranche le seul vers isolé (v. 13 avec «maison»), on obtient structurellement quatre quatrains à rimes croisées.

En nous penchant sur les ajouts manuscrits, on voit tout de suite à quel point la double recherche du huit et de la rime oriente les retouches des premiers vers : dans la première version du poème, «incline» ne rime avec aucune autre fin de vers. En ajoutant «à la lumière», Reverdy obtient un octosyllabe tout en ajoutant un élément qui rime avec «derrière » et «poussière »- deux termes autour desquels il tâtonne manifestement, puisque tous deux sont barrés dans le texte de départ, mais réintroduits ailleurs ${ }^{13}$. Quoi qu'il en soit, on voit bien ici à l'œuvre le procédé qui consiste à remplir les blancs du texte pour combler la mesure de l'octosyllabe, que ce soit à gauche (v. 3) ou à droite (v. 1-2).

\section{Le fond/la forme}

Dans cette opération de comblement, il arrive bien souvent que les préoccupations syntaxiques ou métriques prennent le pas sur le contenu. Rien d'étonnant, certes, à ce que l'anecdote ne soit pas centrale chez un poète qui ne cesse d'affirmer que la poésie ne doit pas imiter le monde mais créer un événement, qu'elle doit présenter plutôt que représenter ${ }^{14}$. En comparant les versions, on est pourtant souvent surpris des changements très marqués dans la nature de l'événement présenté. Citons à titre d'exemple le début de «Visage» (p. 174) : de «Il sait d'où tu viens» à «Il sait à peine d'où tu viens», on passe de cinq à huit syllabes... et on dit presque le contraire! Puis, au vers suivant, le remplacement de «Une ride le marque» par

10. C'est-à-dire celle de Plupart du temps, mais aussi celle des Épaves du ciel, car, comme c'est presque toujours le cas, le matériau verbal ne subit aucune retouche entre le recueil de 1924 et celui de 1945.

11. Voir par exemple l'article «Syntaxe» que Reverdy livre à Nord-Sud en avril 1918, dans Euvres complètes, op. cit., p. 500-501. On peut établir un lien entre cette refonte de la syntaxe et ce que Reverdy admirait vivement chez ses amis les peintres cubistes : un renouvellement du regard impliqué par un agencement des objets susceptible de faire surgir en chacun d'eux ce qu'il a de constant, en dehors de tout ancrage anecdotique, et de faire surgir de leur rencontre, pour le spectateur, des rapports neufs (voir ibid., p. 457-461 ou p. 547-549).

12. L'apocope sur «roues» (v. 8) ne pose plus problème en 1918, et il semble que «il (n') y a » (v. 14) compte toujours deux syllabes chez Reverdy.

13. « qui bout derrière » est ajouté temporairement au vers 3, mais abandonné sur le papillon (et dans la version définitive), tandis que «sous la poussière » est finalement retenu après avoir, semble-t-il, subi un coup de gomme sur la page corrigée au crayon. 
«Malgré la ride qui te marque» porte la ride à changer de visage, et toute l'organisation actancielle de ce microrécit s'en trouve chamboulée. Le cas n'est pas exceptionnel, et il semble bien que le renforcement des liens syntaxiques ne soit pas simplement un moyen de dévoiler quelque chose de préexistant et d'inaltérable, qu'on n'apercevait pas assez dans la première version (par excès de pudeur, suggérait Reverdy). Les déploiements syntaxique et métrique entraînent parfois des modifications nettes de la substance événementielle même, qui peut donc difficilement s'entendre comme l'élément fixe autour duquel se déploieraient les variations formelles.

À ce stade, la nature de l'invariant demeure mystérieuse. S'il y a bel et bien quelque chose qui fonde l'identité de ces textes, des raisons qui invitent à les considérer comme des versions d'un même poème, ce noyau d'identité ne réside donc ni dans le monde construit, ni dans la syntaxe, ni dans la métrique.

Il n'est pas non plus (et c'est peut-être encore plus surprenant) dans le dispositif typographique. Notre poème «Campagne», dans sa première version, se déployait sur six niveaux d'alignement à partir de la marge de gauche. Il correspondait, comme l'ensemble des poèmes de cette édition originale, à l'idée de doter la page d'un rythme pour l'œil, comme Reverdy l'indiquait dans Self defence. Or dans la version définitive, tout est purement et simplement ferré à gauche. Rien ne subsiste de la disposition initiale, dont il faut conclure qu' au moment de la réécriture, elle apparaissait à Reverdy comme inutile ou superflue. Si cet alignement généralisé à la marge de gauche est relativement fréquent dans le recueil, il ne constitue pourtant pas une norme : la plupart des poèmes des Ardoises conservent un certain nombre de décrochements en débuts de vers, mais la quantité de ceux-ci a très nettement diminué par rapport à l'édition de 1918.

Mais le plus surprenant, au vu de l'exemplaire annoté par Reverdy, c'est l'absence totale de marquage explicite de la disposition spatiale des vers sur la page. Ce Reverdy, correcteur professionnel - ce Reverdy qui avait, avec sa femme Henriette, soigné attentivement la réalisation typographique de l'édition originale des Ardoises - le voilà qui révise ses poèmes sans accorder la moindre valeur spatiale à son geste de réécriture : à aucun moment, la position du trait sur la page ne semble valoir pour une instruction concernant sa position dans l'édition à venir.
Faudrait-il en conclure que la mise en page de l'édition originale n'était qu'une coquetterie, ou en tout cas un élément accessoire, secondaire ? On peine à croire par exemple que les premiers vers de «Manchette» (p. 170) aient pu être rédigés sans l'idée conjointe de leur disposition. Mise en texte et mise en page semblaient, ici au moins, indissociables ${ }^{15}$. Quoi qu'il en soit des étapes antérieures, au stade de la genèse post-éditoriale du texte, la mise en espace et la mise en mots sont des processus dissociés. Sur le livre corrigé, aucune instruction topographique n'accompagne la textualisation. Par exemple, les corrections au crayon de «Visage» apparaissent là où la place le permet, sans qu'on puisse en inférer précisément la position que devront occuper les vers dans la prochaine édition. Cette position a dû être fixée plus tard, sur épreuves peut-être.

De sa première manière, Reverdy ne s'est pas seulement éloigné dès les années vingt, il l'a partiellement éliminée après coup des recueils qui la représentaient le mieux. Les Ardoises du toit est-il encore un texte de 1918, comme l'annoncent la page de titre et la table des matières des Épaves du ciel et de Plupart du temps? On répondra à cette question en disant que si l'objet graphique, le livre, a une date de publication, l'énonciation écrite combine les âges et les sujets successifs de son élaboration.

Une analyse de détail reste à entreprendre, mais il y a lieu de penser que le document qu'on lira ici témoigne d'un parcours étonnant où la poétique d'une œuvre, telle qu'elle a été reconnue et célébrée par la critique, n'est pas située à la fin de son élaboration ${ }^{16}$, mais à une étape antérieure. C'est l'esthétique d'une étape antérieure, mais publique, celle de la première publication du recueil, qui a fait date et histoire.

14. Reverdy l'exprime notamment dans une note qu'il publie dans Nord-Sud à propos des Ardoises du toit. Sous le pseudonyme de S. Laforêt, il écrit : «Chaque poème est ici un fait poétique présenté au lieu d'être une anecdote représentée. L'art de M. Reverdy est un art simple de présentation, de création - qui n'existait pas avant lui » (Euvres complètes, op. cit., p. 505).

15. Plus précisément dit, la structuration segmentale (morphosyntaxique) semble fortement dépendante de la structuration topographique (fait de ponctuation et de disposition), ces deux ordres de signifiance intégrant toujours et partout l'énonciation écrite.

16. Comme dans l'exemple archétypique de l'évolution du premier conte d'Andersen au bout de laquelle celui-ci a trouvé sa formule générique (voir Cyrille François ici même, p. 65) 
Visite' (crayon sur l'édition, ci-contre)

Les bateaux s'étageaient dans le tableau du fond Les hommes qui jouaient aux cartes

Les rideaux immobiles

Et les mots qui s'en vont

Devant eux [1]a fumée s'écarte

Un livre a refermé ses portes

La prison des pensées où la mienne était morte

Toutes les bouches qui riront

gagneront la fenêtre et l'air [s]ur le balcon

Les vitres d'en face pâlissent

Visite" (mise au net à l'encre noire, ci-dessous)

Les bateaux s'étageaient dans le tableau du fond Où les hommes jouaient aux cartes

Les mots les plus légers montent jusqu'au plafond Devant eux la fumée s'écarte

Les autres battent des ailes dans les plis des rideaux

L'ennui de la soirée pèse sur les cerveaux

Un livre a refermé ses portes

La prison des pensées où la mienne était morte

Toutes les bouches qui riront

gagneront la fenêtre et l'air sur le balcon

Les vitres d'en face pâlissent
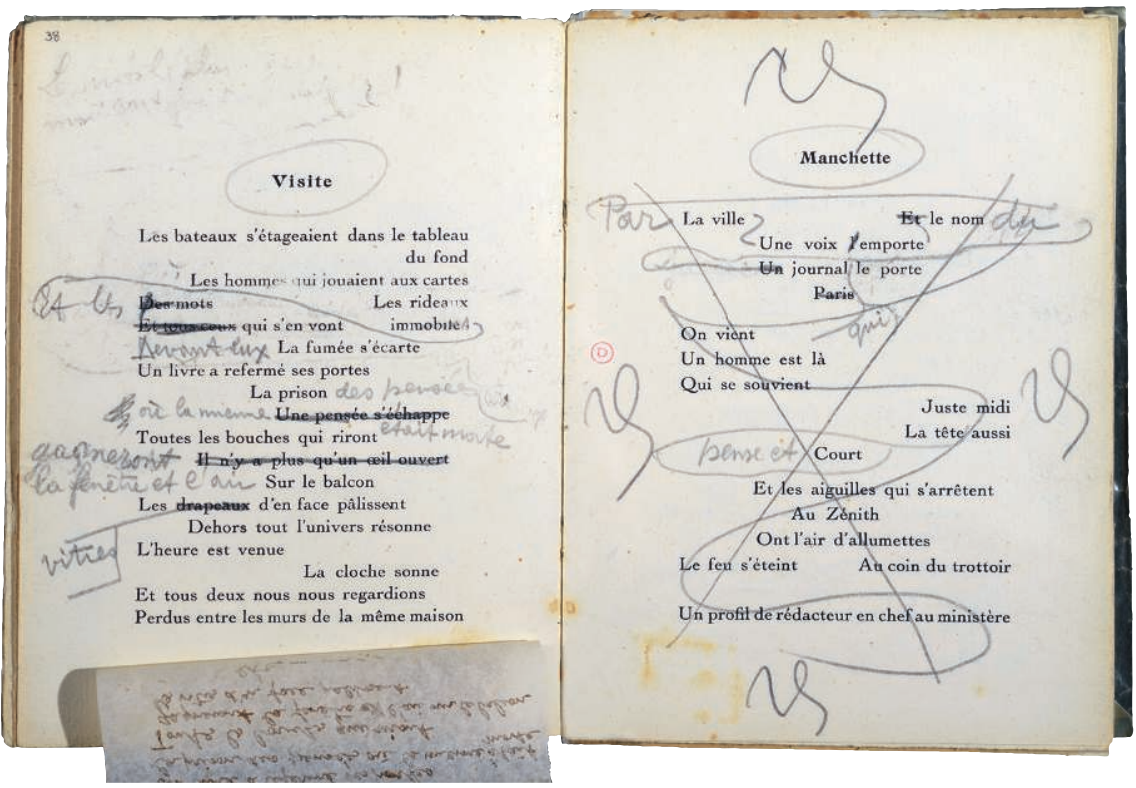

À droite, le seul poème définitivement supprimé de l'édition originale. On mesurera l'écart entre la version originale du poème et le début de sa réécriture qui donnait : «Par la ville une voix emporte le nom du journal qui le porte»

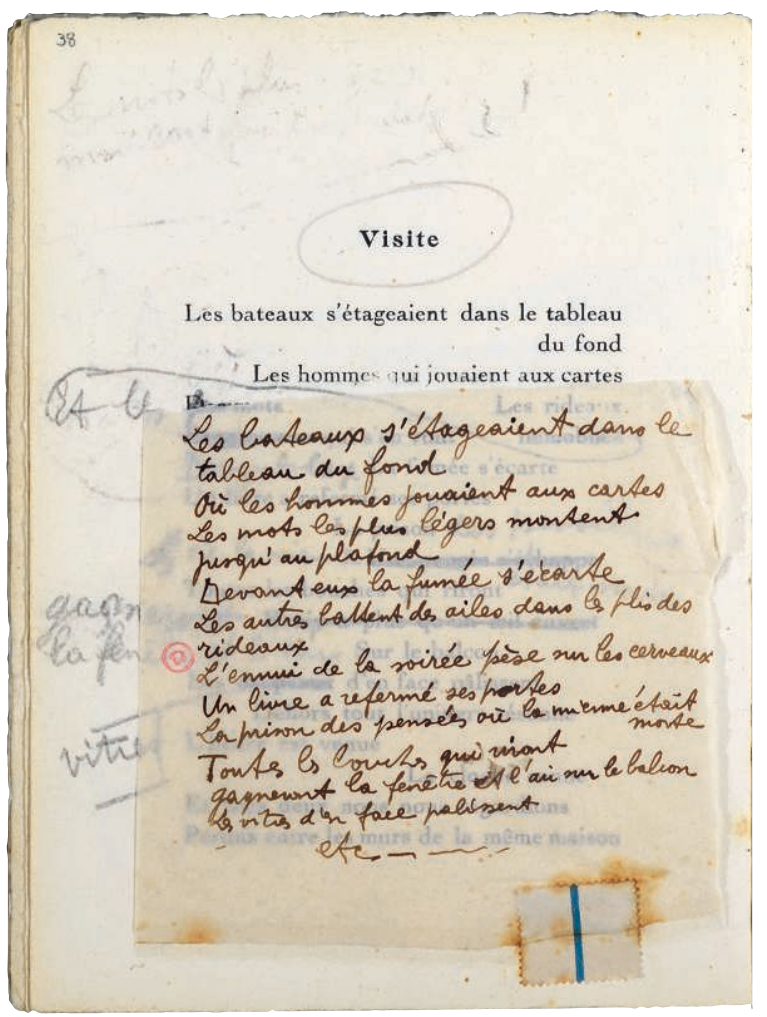

La mise au net de «Visite», sur le collage, n'est pas la suite directe de sa révision au crayon; entre les deux versions du poème, la comparaison textuelle dégage des suppressions, des ajouts et un déplacement dont le document ne porte pas traces.

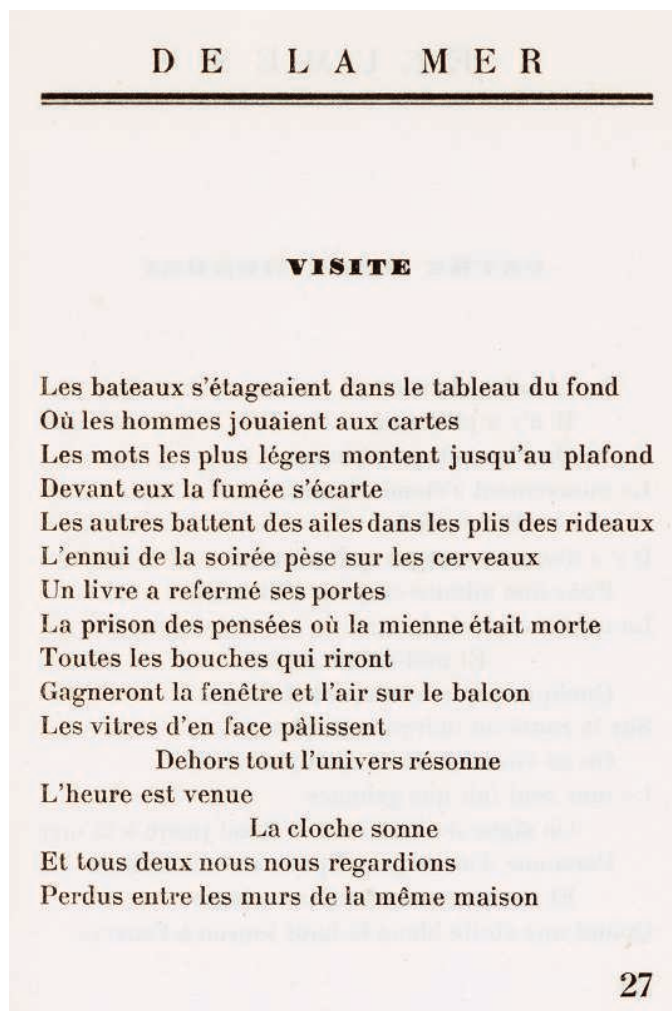

Écumes de la mer adopte la version définitive de l'originale corrigée. Les deux seuls décrochages de vers qui subsistent apparaissent moins comme un choix que comme un vestige de l'édition originale dont la fin n'a pas été réécrite. Le premier de ces décrochages («Dehors tout l'univers...») disparaît dans Plupart du temps. 


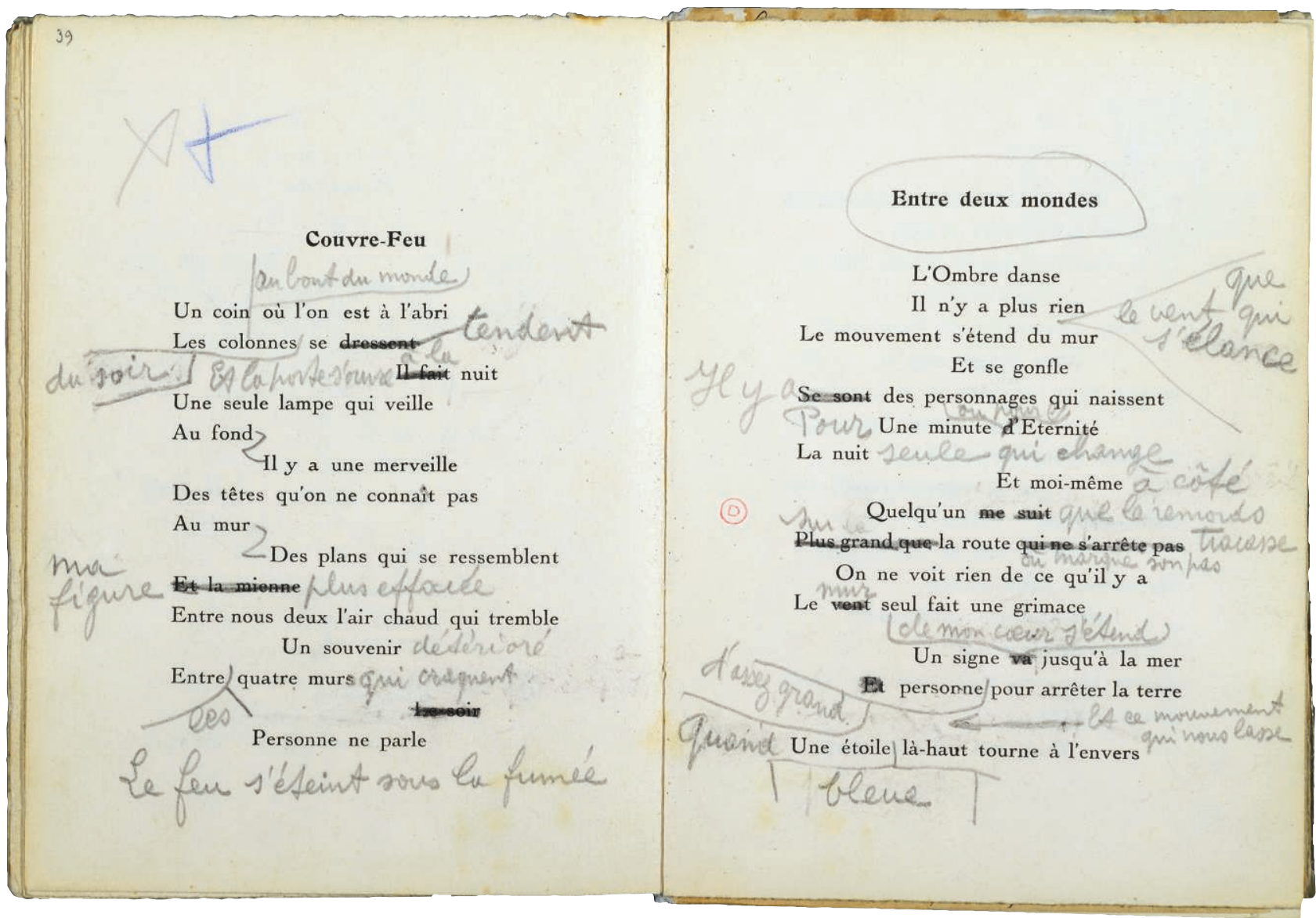

«Couvre-Feu » illustre bien les deux tendances qui caractérisent la révision des poèmes pour Les Épaves du ciel. La «chair» verbale ajoutée à la version originale du poème construit un référent plus net et circonstancié ; en même temps, les ajouts et les $Z$ alignant les vers en créneau régularisent la métrique. Désormais, onze vers (sur treize) sont octosyllabiques (vs quatre sur quinze dans l'édition originale) et seul un vers inférieur à huit syllabes subsiste («Personne ne parle»), alors que l'édition originale en comptait onze. Cette dernière confiait à l'espace un pouvoir d'organisation et de mise en relation propre; l'édition de 1924 rabat l'espace graphique presque entièrement sur la structure syllabique du poème.

«Entre deux mondes», qui sera repris dans Écumes de la mer (1925), conserve plus de variétés topographiques. Plupart du temps, qui reproduira littéralement la version de 1925, ne rabattra rien de sa variété spatiale, tout en présentant plusieurs légères différences de disposition.

\section{COUVRE-FEU}

Un coin au bout du monde où l'on est à l'abri Les colonnes du soir se tendent

Et la porte s'ouvre à la nuit

Une seule lampe qui veille

$\mathrm{Au}$ fond il y a une merveille

Des têtes qu'on ne connaît pas

Au mur des plans qui se ressemblent

Ma figure plus effacée

Entre nous deux l'air chaud qui tremble

Un souvenir détérioré

Entre les quatre murs qui craquent

Personne ne parle

Le feu s'éteint sous la fumée

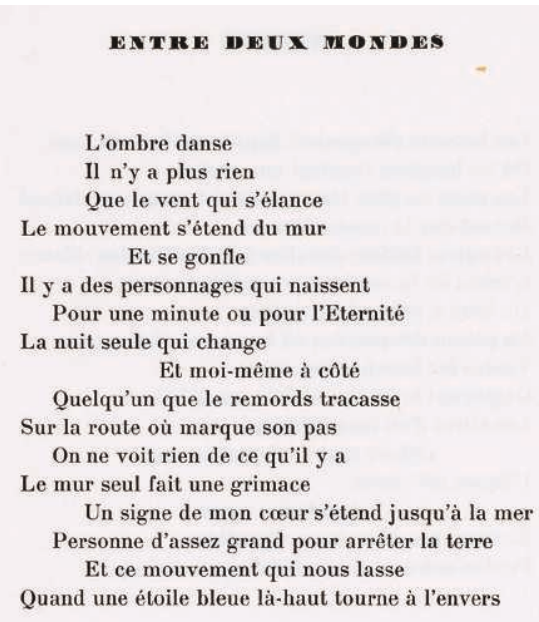

28 


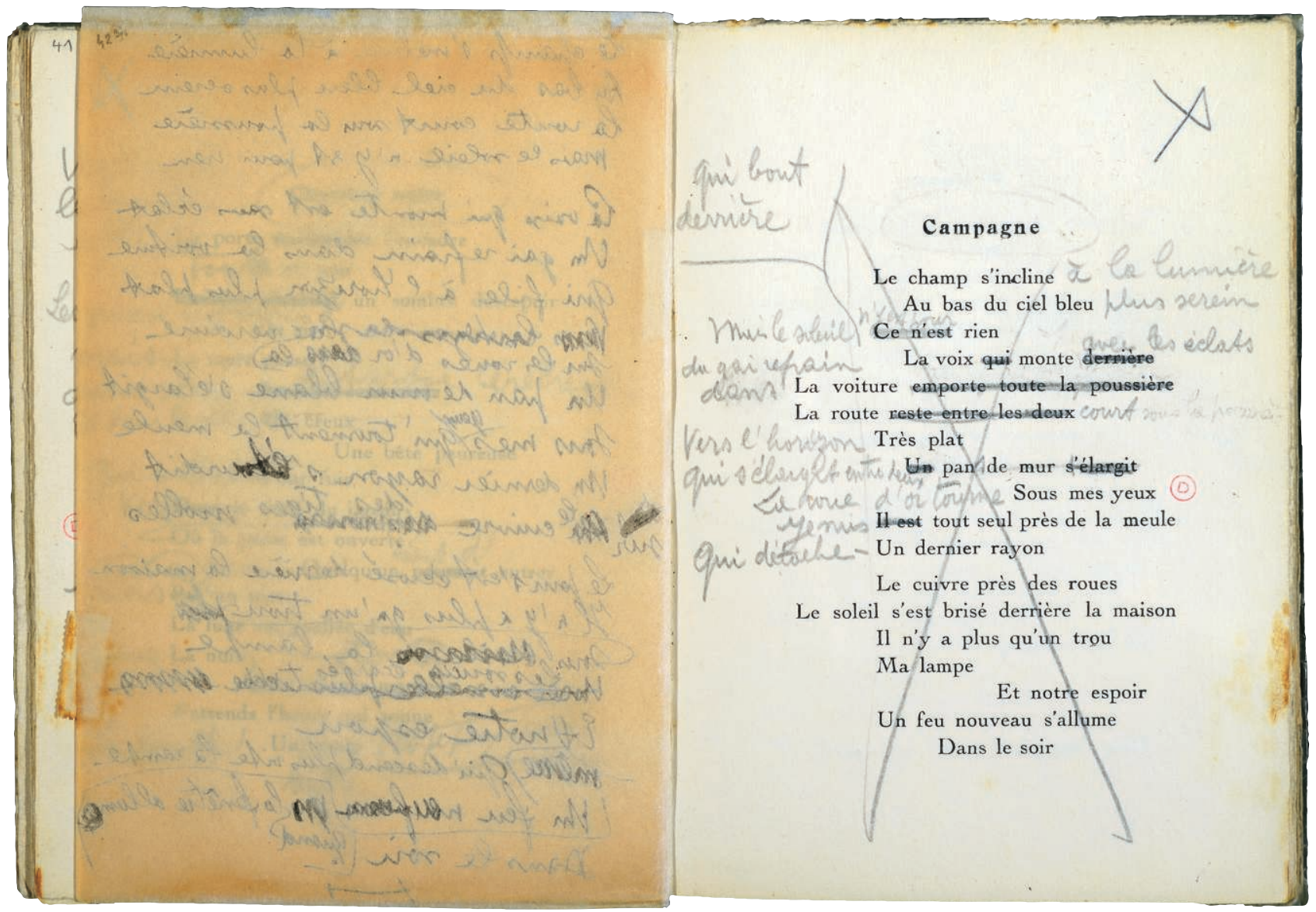

Transcription des vers 4-11:

a) sur la page imprimée retouchée, ci-dessus : «La voix monte avec les éclats / du gai refrain dans la voiture / La route court sous la poussière / Vers l'horizon très plat / Qui s'élargit entre deux pans de mur / La roue d'or tourne sous mes yeux / Je suis tout seul près de la meule / Qui détache un dernier rayon ».

b) sur le papillon, ci-dessous : «La voix qui monte est sans éclat / Un gai refrain dans la voiture / Qui file à l'horizon plus plat / Vers les tiges de la Sur les roues d'or dans la verdure / Un pan de mur blanc s'élargit / Sous mes yeux qui tournent la meule / Un dernier rayon s'alourdit s'étourdit / Au Sur le cuivre des roues des tiges molles ». 


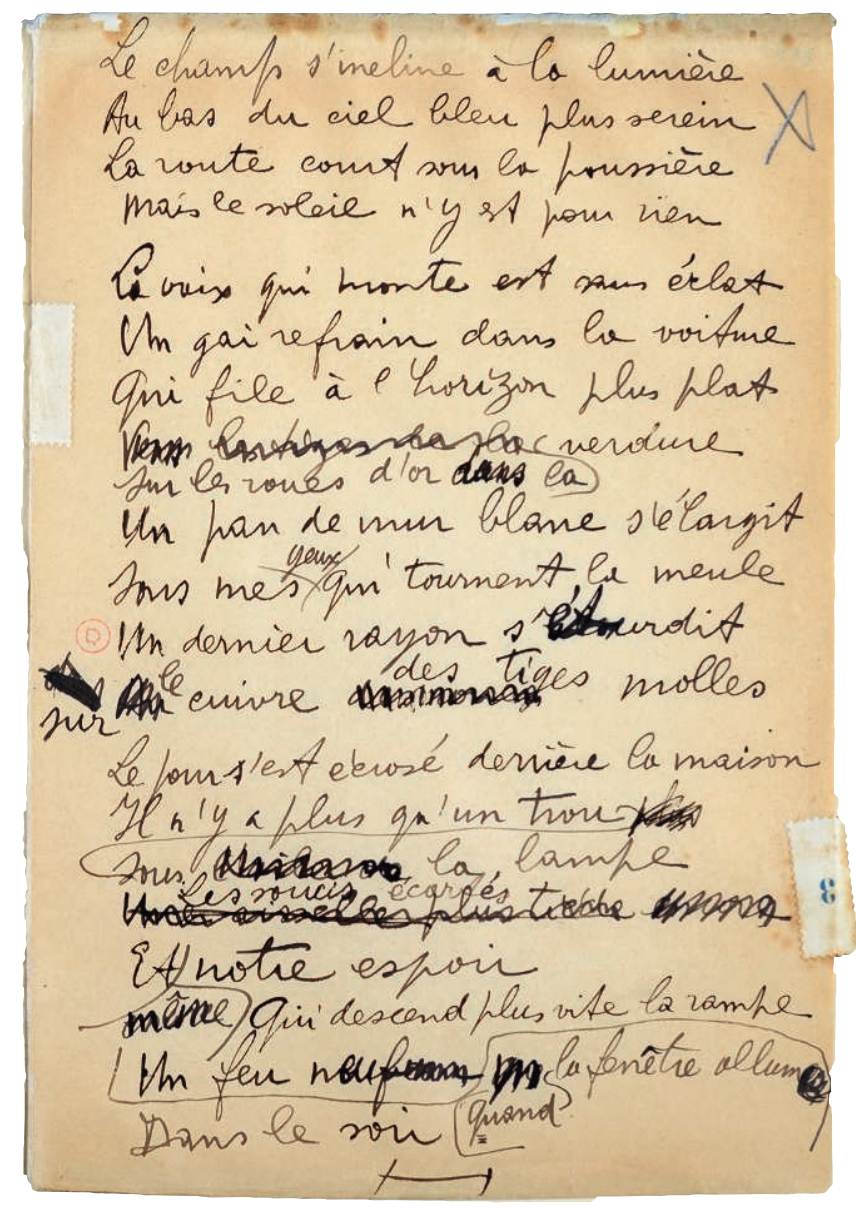

LES ÉPAVES DU CIEL

\section{GAMPAGNE}

Le champ s'incline à la lumière Au bas du ciel bleu plus serein La route court sous la poussière Mais le soleil n'y est pour rien

La voix qui monte est sans éclat Un gai refrain dans la voiture Qui file à l'horizon plus plat Sur les roues d'or dans la verdure Un pan de mur blane s'élargit Sous mes yeux qui tournent la meule Un dernier rayon s'étourdit Sur le cuivre des tiges molles

Le jour s'est écrasé derrière la maison Il n'y a plus qu'un trou sous la lampe Les soucis écartés et même notre espoir Qui descend plus vite la rampe Quand la fenêtre allume un feu neuf dans le soir

Pour ce poème, le matériau génétique est particulièrement riche. Les retouches inscrites au crayon forment une première strate du processus de réécriture; elles occupent vite tout l'espace marginal à disposition, si bien que Reverdy s'interrompt après le vers 11 pour retranscrire le texte sur un papillon séparé. Lors du passage sur ce nouveau support, le poète modifie considérablement son texte, produisant ainsi un nouvel état du texte. Le papillon est retouché à son tour, de plus en plus massivement au fil des vers; ces retouches constituent une nouvelle strate, elle-même multiple, puisqu'on y observe quelques états transitoires (ainsi, aux deux tiers du feuillet, «dans le», préféré à «au » avant d'être remplacé par «sur le»). Parmi ces variantes abandonnées, relevons un vers supprimé peu avant la fin, qui semble être «Une aisselle plus tiède encore ». Quant au dernier vers, il accrédite l'idée que l'élaboration du poème ne s'achève pas sur le papillon : «Dans le soir» constitue ici un dernier vers, isolé, alors que le texte des Épaves s'achève sur un alexandrin qu'aucune des strates considérées ne laissait attendre. 

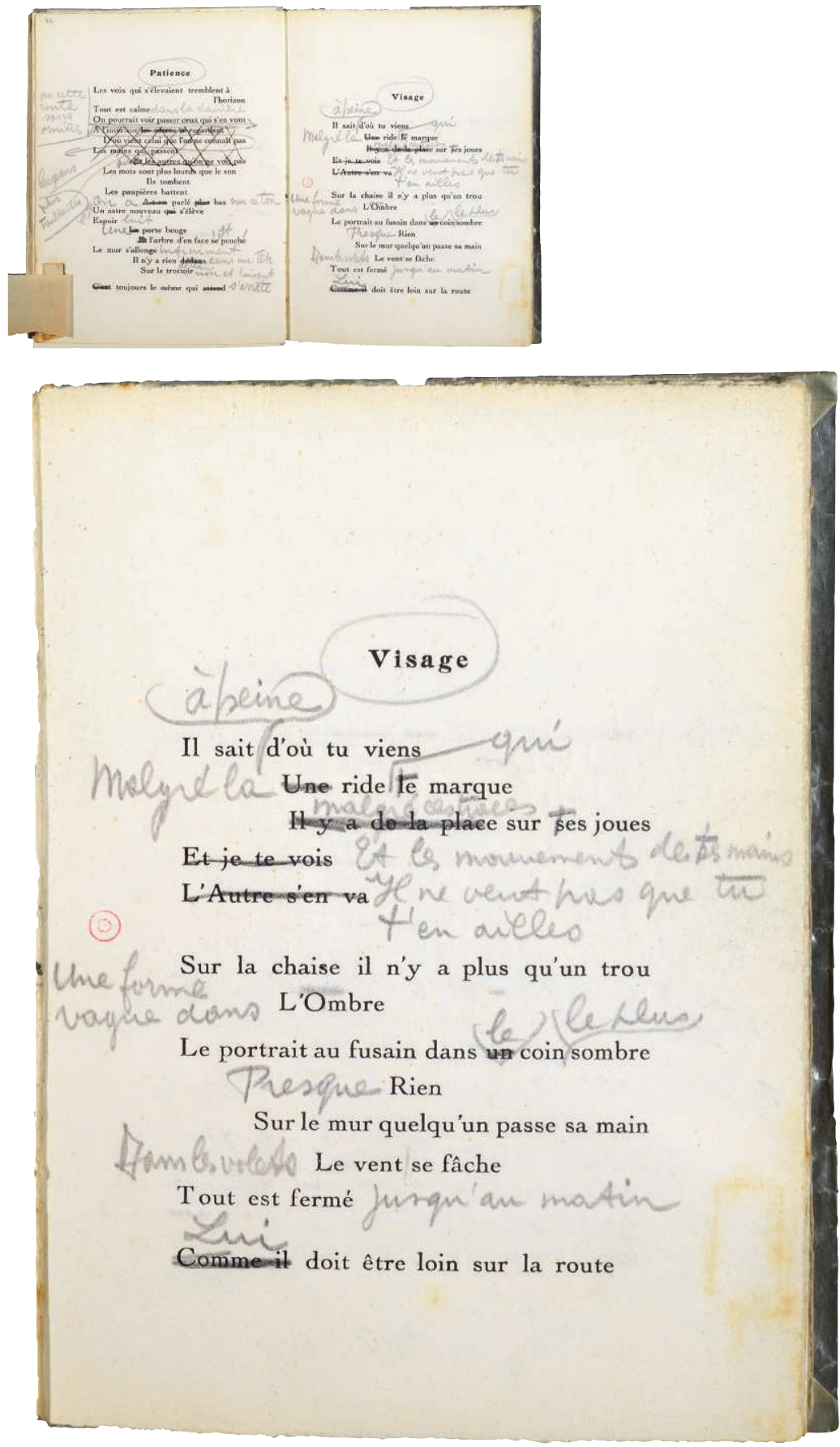

É C U M E S

\begin{tabular}{l}
\hline \hline VIS A GE \\
Il sait à peine d'où tu viens \\
Malgré la ride qui te marque \\
Malgré ces traces sur tes joues \\
Et les mouvements de tes mains \\
Il ne veut pas que tu t'en ailles \\
Sur la chaise il n'y a plus qu'un trou \\
Une forme vague dans l'ombre \\
Le portrait au fusain dans le coin le plus sombre \\
Presque rien \\
Sur le mur quelqu'un passe sa main \\
Dans les volets le vent se fàche \\
Tout est fermé jusqu'au matin
\end{tabular}

Lui doit être loin sur la route

32

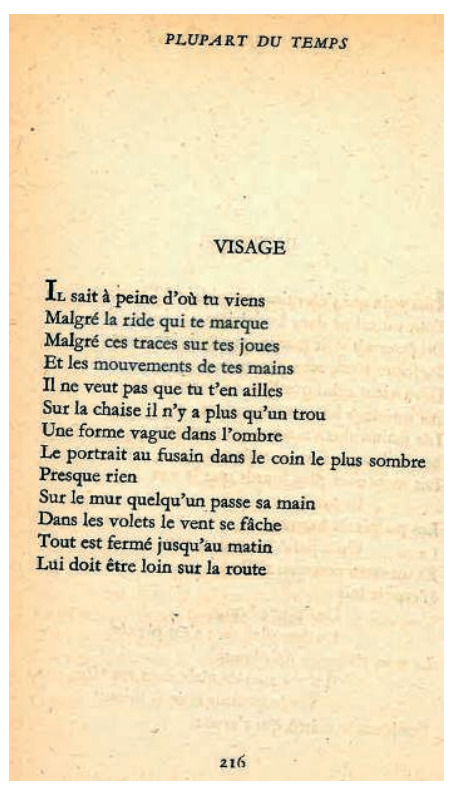

«Visage» présente un exemple frappant d'assagissement formel. Ce poème fait en outre exception dans le corpus considéré ici, du fait que cette standardisation se produit en deux étapes bien distinctes : le texte de 1945 (Plupart du temps) est lexicalement et métriquement identique à celui de 1925 (Écumes de mer), mais il est très différent sur le plan topographique. On observe ainsi d'abord une régularisation métrique, qui privilégie l'octosyllabe de façon spectaculaire : la version de 1918 n'en présente qu'un seul (pour autant que l'on compte «il y a» comme deux syllabes - voir ci-dessus note 13); dans Écumes de la mer, ce chiffre est décuplé. Mais la disposition spatiale est aussi variée d'un côté que de l'autre, avec quatre ou cinq niveaux d'alignement. Il est cependant intéressant de noter que l'agencement des décalages latéraux est tout à fait différent d'une version à l'autre : les vers alignés ne sont plus les mêmes, et les décrochements passent librement de droite à gauche, ou de gauche à droite. À cette diversité peu canalisée, la version de Plupart du temps réserve, dans un second temps, un traitement radical : l'alignement généralisé à la marge de gauche. 


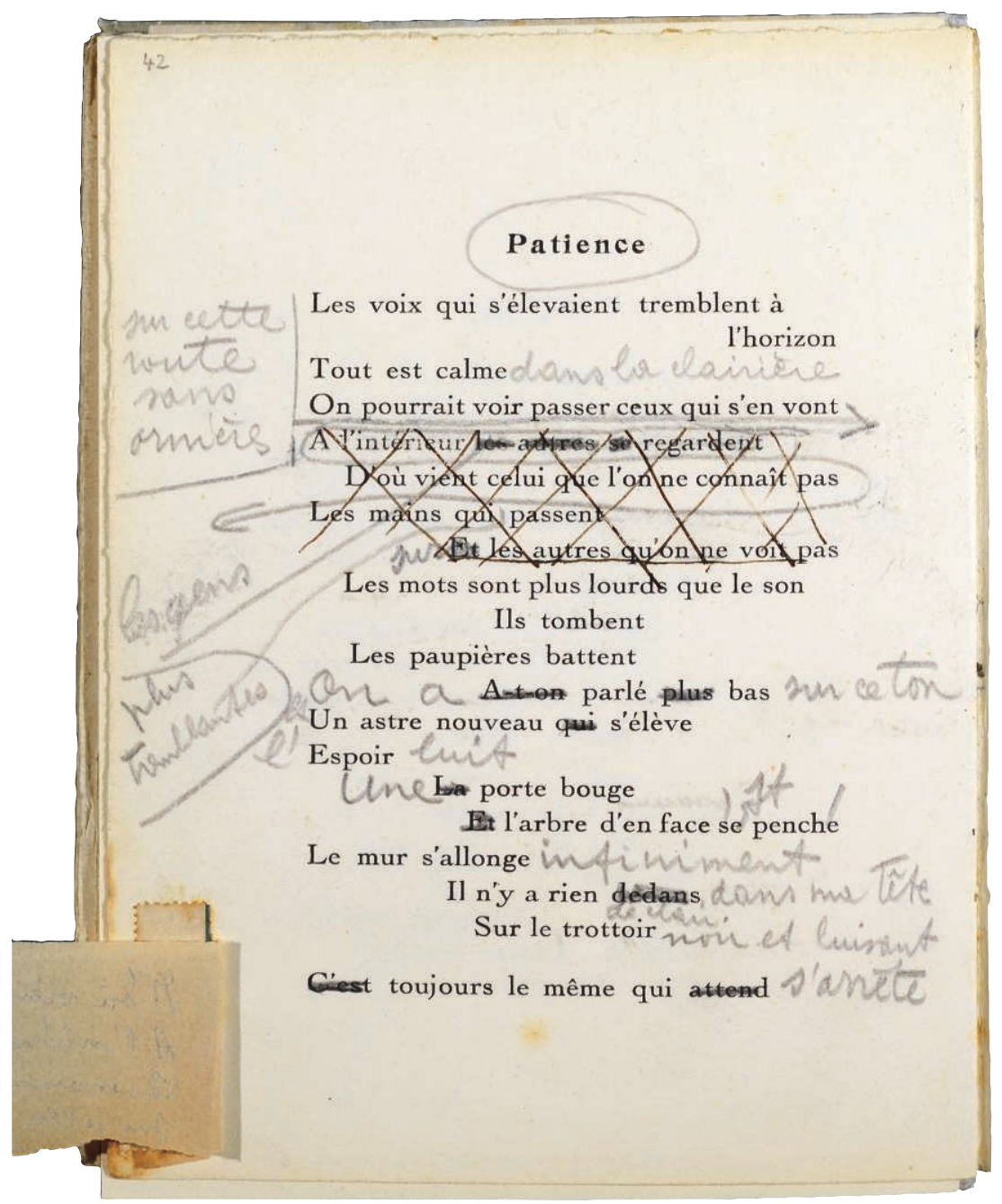

É C U M E S

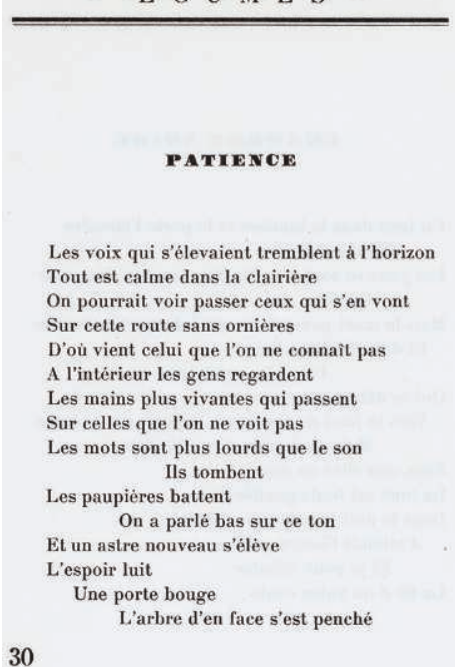

30

\section{E L A M E R}

Le mur s'allonge infiniment II n'y a rien de clair dans ma tête Sur le trottoir noir et luisant Toujours le même qui s'arrête
Face à la gradation marquée qui caractérise «Visage», «Patience» propose un cas beaucoup plus modeste : les décrochements sont un peu différents d'une version à l'autre, mais le dispositif spatial d'ensemble demeure. Qu'est-ce qui pouvait laisser prévoir que «Visage» perdrait dans l'édition définitive les subtilités topographiques que «Patience» conserverait dans une large mesure?

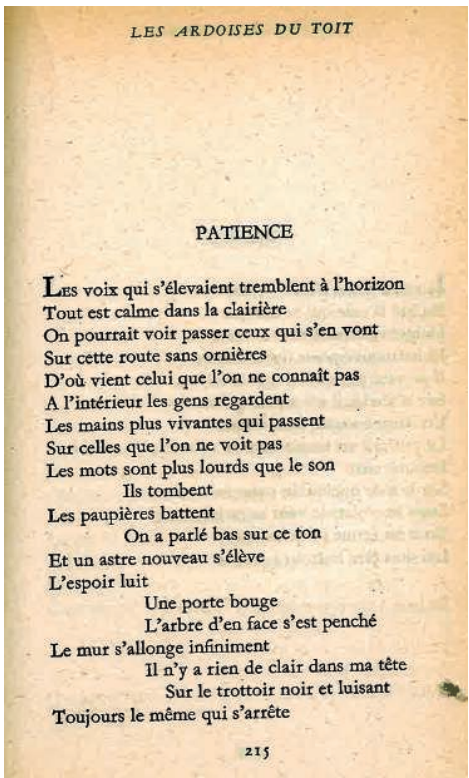



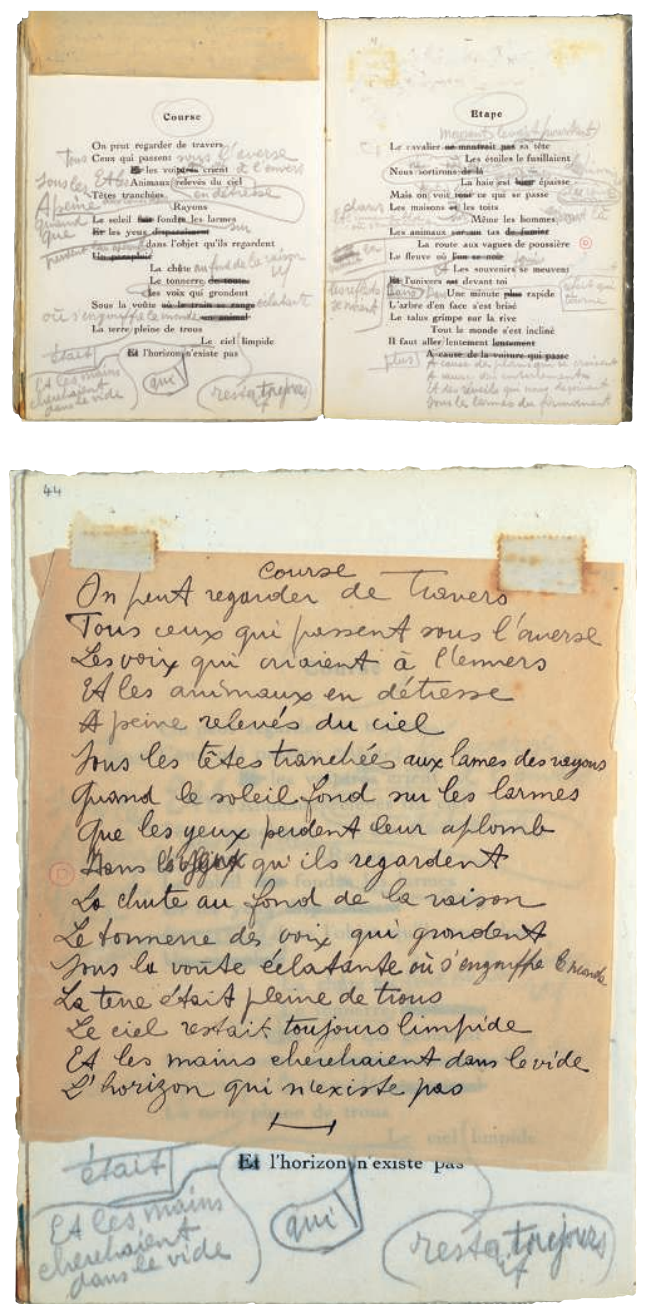

\section{$\operatorname{Cotrse}$}

On peut regarder de travers Tous ceux qui passent sous l'averse Les voix qui criaient à l'envers Et les animaux en détresse A peine relevés du ciel

Sous les têtes tranchées aux lames des rayons Quand le soleil fond sur les larmes Que les yeux perdent leur aplomb Dans les yeux qu'ils regardent La chute au fond de la raison Le tonnerre des voix qui grondent Sous la voûte éclatante où s'engouffre le monde La terre était pleine de trous

Le ciel restait toujours limpide Et les mains cherchaient dans le vide L'horizon qui n'existe pas

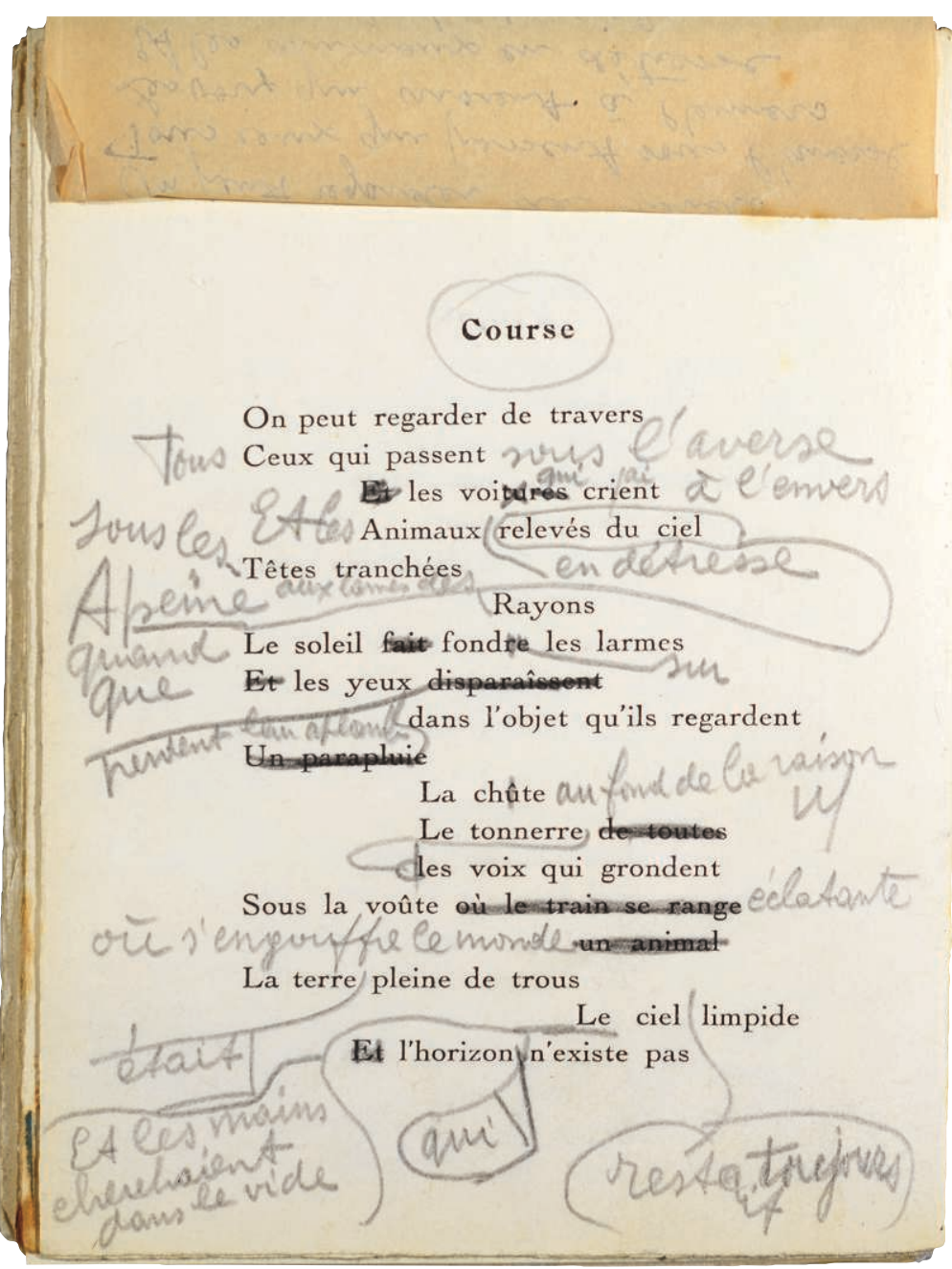

Contrairement à ce qui se passe pour «Visite», le texte du papillon collé sur «Course» est une stricte mise au net de la réécriture du poème au crayon. On y observe une seule rature («objet» $\rightarrow$ «yeux», vers 10), cherchant la répétition (des «yeux», dans un face à face) et évitant un vers impair qui répugne au Reverdy du début des années 1920. Si la mise au net à la plume semble d'abord motivée par la quantité des interventions au crayon, elle est aussi l'occasion de débarrasser le texte de toute variation topographique : le poème passé par la plume est ferré à gauche. Sa réédition dans Écumes de la mer puis dans Plupart du temps entérine cette disposition. 


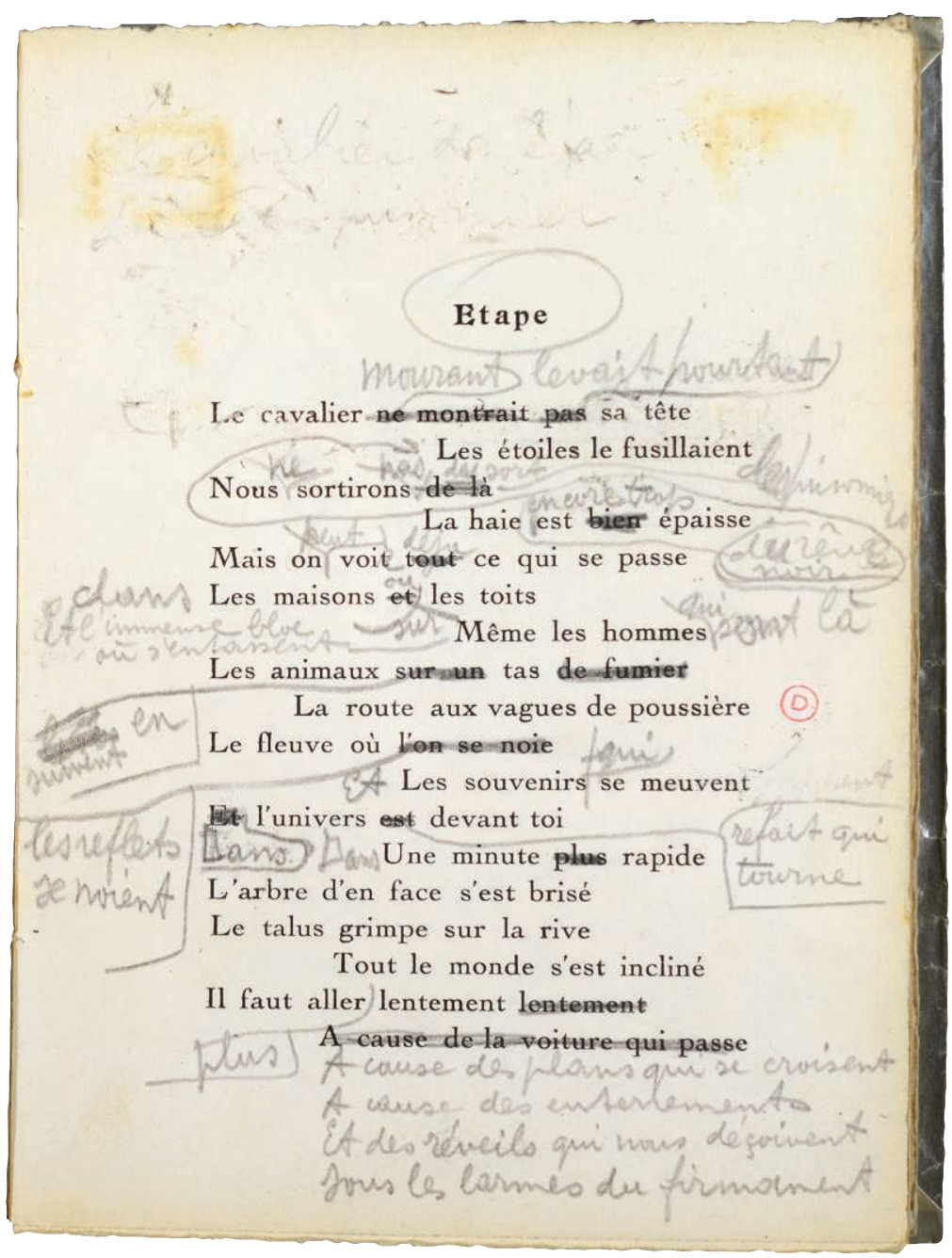

«Étape», qui n'a pas connu de mise au net à la plume en 1918, conserve, dans l'édition de 1925, quelques-uns de ses «créneaux» d'origine, bien que rapetissés ou comblés par des mots insérés.

Les vers ajoutés à la fin sont même alignés sur le dernier vers de l'édition originale (il est difficile de l'apprécier dans l'édition de 1925, mais cela est manifeste dans celle de 1945).

É C U M E S

ETAP E

Le cavalier mourant levait pourtant sa tête Les étoiles le fusillaient La haie du rêve noir est encore trop épaisse Nous ne sortirons pas du sort des prisonniers Mais on peut voir déjà ce qui se passe Dans les maisons ou sur les toits Et l'immense bloc où s'entassent Même les hommes qui sont là

Les animaux suivent en tas

La route aux vagues de poussière

Le fleuve oú les reflets se noient

Et les souvenirs qui se meuvent

Dans l'univers refait qui tourne devant toi Dans une minute rapide

L'arbre d'en face s'est brisé

Le talus grimpe sur la rive Tout le monde s'est incline

Il faut aller plus lentement

\section{E L A M E R}

A cause des plans qui se croisent A cause des enterrements

Et des réveils qui nous déçoivent

Sous les larmes du firmament 


\section{Ecran}

Une ombre coule sur ta main La lampe a changé ta figure La pendule bat Le temps dure

Et comme il ne se passe rien Celui qui regardait s'en va

Le monde se retourne et rit Pour regarder tout ce qui vit

On marche envine dema 420

Un tournant au bout de la route

Un pont sans arches

$$
\text { Une forêt }
$$

Et la maison où je vivrais

Il faut partir coûte que coûte

Et l'ombre qui passait

Le monde qui riait

$$
\text { Celui qui regardait }
$$

Au fond contre le mur

$$
\text { S'évanouissent }
$$

Des silhouettes glissent
Et $1 a ̀$

Quelqu'un parle et je suis debout

Je vais partir là-bas à l'autre bout Les arbres pleurent

$\mathrm{Au}$ loin où d'autres choses meurent mhimplenu La tête a tout pris

Je ne t'ai pas encore compris

On marche

Passer par une porte où personne n'attend Thu th Impossible repos

Tout s'écarte et montre le dos

$\mathrm{Ou}$ Un peu de vide autour

Revivre d'anciens jours

Une âme qui s'amuse

Et traîne un corps qui s'use

Le dernier temps une mesure

Plus Lonace Par là

Un chagrin musical murmure

Comme on l'a vu, le livre corrigé constitue l'ultime moment de l'élaboration du texte des Ardoises du toit. Cette observation est vraie en ce qui concerne la facture verbale des poèmes; en revanche, leur dimension topographique reste encore souvent à préciser. L'absence d'instructions concernant la disposition des vers frappe, en effet, à la lecture des pages de ce livre redevenu brouillon. Par exemple, dans «Et là», pourquoi le vers 5 ( «Maintenant la tête a tout pris ») n'a-t-il pas été ferré à gauche dans Les Épaves du ciel, alors que le vers 12 («Et pour revivre d'anciens jours») l'a été? Ou pour prendre un cas plus frappant, quid de la ligne blanche qui apparaît dans l'édition de 1924 après le huitième vers d' «Écran» et le cinquième d' «Et là», ou encore, dans ce dernier poème, du léger retrait des vers 1 et 10 ? L'exemplaire corrigé n'en dit rien.

On observe ainsi que les plans segmentaux et suprasegmentaux ont fait l'objet d'une révision partiellement dissociée; c'est seulement après avoir transformé leur «grammaire», sur l'exemplaire corrigé, que Reverdy aménage le nouvel espace de ses poèmes (vraisemblablement sur les épreuves des éditions de 1924 et de 1945). 
104

\section{LES ÉPAVES DU CIEL \\ ECRAN}

Une ombre coule sur ta main La lampe a changé ta figure

Le pendule bat

Le temps dure

Et comme il ne se passe rien

Celui qui regardait s'en va

Le monde se retourne et rit

Pour regarder tout ce qui vit

On marche encore dans le doute

Un tournant au bout de la route

Une forêt

Un pont sans arches

Et la maison où je vivrais

Il faut partir coûte que coûte

Et l'ombre qui passait

Le monde qui riait

Celui qui regardait

Au fond contre le mur

S'évanouissent

Des silhouettes glissent
116

LES ÉPAVES DU CIEL

ET LA

Quelqu'un parle et je suis debout

Je vais partir là-bas à l'autre bout

Les arbres pleurent

Parce qu'au loin d'autres choses meurent

Maintenant la tête a tout pris

Mais je ne t'ai pas encore compris

Je marche sur tes pas sans savoir qui je suis

Il faut passer par une porte où personne n'attend

$$
\text { Pour un impossible repos }
$$

Tout s'écarte et montre le dos

Un peu de vide reste autour

Et pour revivre d'anciens jours

Une âme détachée s'amuse

Et traîne encore un corps qui s'use

Le dernier temps d'une mesure

Plus tenace et plus déchirant

Un chagrin musical murmure

\section{LES ARDOISES DU TOII}

\section{ÉCRAN}

$\mathrm{U}_{\mathrm{NE}}$ ombre coule sur ta main La lampe a changé ta figure La pendule bat

\section{Le temps dure}

Et comme il ne se passe rien

Celui qui regardait s'en va

Le monde se retourne et rit

Pour regarder tout ce qui vit

On marche encore dans le doute

Un tournant au bout de la route

Un pont sans arches

$$
\text { Une forêt }
$$

Et la maison où je vivrais

Il faut partir coûte que coûte

Et l'ombre qui passait

Le monde qui riait

$$
\text { Celui qui regardait }
$$

$\mathrm{Au}$ fond contre le mur

$$
\text { S'évanouissent }
$$

Des silhouettes glissent

\section{PLUPART DU TEMPS}

\section{ET LA}

Quelou'un parle et je suis debout Je vais partir là-bas à l'autre bout Les arbres pleurent

Parce qu'au loin d'autres choses meutent Maintenant la tête a tout pris

Mais je ne t'ai pas encore compris Je marche sur tes pas sans savoir qui je suis Il faut passer par une porte où personne n'attend Pour un impossible repos

Tout s'écarte et montre le dos

Un peu de vide reste autour

Et pour revivre d'anciens jours

Une âme détachée s'amuse

Et traîne encore un corps qui s'use

Le dernier temps d'une mesure

Plus tenace et plus déchirant

Un chagrin musical murmure 
CHRISTOPHE IMPERIALI est chercheur auprès du Fonds national suisse de la recherche scientifique, rattaché à l'université de Berne. Après une thèse portant sur le mythe de Perceval dans la littérature, il s'oriente davantage vers la poésie des XIX ${ }^{\mathrm{e}}$ et XX $\mathrm{X}^{\mathrm{e}}$ siècles. Il s'intéresse en particulier aux rapports entre poésie et musique, et à ceux que ces deux arts entretiennent avec l'écoulement du temps. christophe.imperiali@rom.unibe.ch

RUdOLF MAHRER est maître-assistant en linguistique française à l'Université de Lausanne et collaborateur de la revue Genesis. Spécialiste de l'écrit et de l'écriture, il vient de publier Phonographie, une étude des modalités de la représentation écrite de l'oral (De Gruyter, 2017). Ses travaux, inscrits dans la tradition de la linguistique de l'énonciation, visent à décrire l'activité de production et d'interprétation des locuteurs et des scripteurs, en accordant une attention particulière aux différences entre énonciation orale et énonciation écrite.

rudolf.mahrer@unil.ch 